\title{
The generation of dense cores and substructure within them by MHD waves
}

\author{
S. Van Loo, S. A. E. G. Falle and T. W. Hartquist \\ University of Leeds, Leeds LS9 JT, UK \\ email: svenvl@ast.leeds.ac.uk
}

\begin{abstract}
By using 2D simulations, we examine the generation of dense cores and substructures by magnetosonic waves. We find that the excitation of slow-mode waves by fast-mode waves produces these high-density structures.
\end{abstract}

Keywords. MHD, stars: formation, ISM: clouds

There have been a number of 3D simulations (e.g. Vázquez-Semadeni et al. 2005) on the effect of MHD waves on an isothermal plasma for which the ratio $\beta$ of thermal to magnetic pressure is small. These simulations show that density inhomogeneities can be formed in this way with statistical properties that are consistent with the observations. However, these calculations generally contain so many ingredients that the fundamental mechanism responsible for the clumpiness of star forming regions is unclear.

The analysis of Falle \& Hartquist (2002) shows that the production of large density contrasts in a low- $\beta$ plasma results when slow-mode waves are excited by the non-linear steepening of a fast-mode wave. Their 1D calculations show that this mechanism can produce transient high-density clumps if gravitational instability does not occur. We have extended this model to 2D (Van Loo, Falle \& Hartquist 2006a).

Like Falle \& Hartquist, we find that the formation of dense structures is associated with slow-mode waves. These slow-mode waves are excited either by the non-linear steepening of the fast-mode wave or by the interaction between the fast-mode wave and dense structures. The latter produces dense cores which reside in larger clumps. The process of producing structure by means of slow-mode waves thus works on different length-scales, but ceases, however, to be effective when $\beta$ approaches unity.

This directly leads to the question of whether substructure in cold dense cores is produced by the same mechanism. Dense cores, however, may have values of $\beta$ only as low as 0.1 (Ward-Thompson 2002). By examining the response of a pre-existing dense core to a fast-mode wave (Van Loo, Falle \& Hartquist 2006b), we find that waves, with wavelengths shorter than the core radius, excite slow-mode waves within the core and, although $\beta$ is close to unity, these slow-mode waves still produce high-density contrasts.

\section{Acknowledgements}

SVL gratefully thanks PPARC for financial support.

\section{References}

Falle, S. A. E. G. \& Hartquist, T. W. 2002, MNRAS 329, 195

Van Loo, S., Falle, S. A. E. G. \& Hartquist, T. W. 2006a, MNRAS 370, 975

Van Loo, S., Falle, S. A. E. G. \& Hartquist, T. W. 2006b, MNRAS, submitted

Vázquez-Semadeni E., Kim J., Shadmehri M., \& Ballesteros-Paredes J. 2005, ApJ 618, 344

Ward-Thompson D. 2002, Science 295, 76 